\title{
PRIMITIVE SKEW LAURENT POLYNOMIAL RINGS
}

\author{
by D. A. JORDAN
}

(Received 27 August, 1976)

Introduction. In [8] the author studied the question of the primitivity of an Ore extension $R[x, \delta]$, where $\delta$ is a derivation of the ring $R$. If $\alpha$ is an automorphism of $R$ then it can be shown that $R[x, \alpha]$ is primitive if the following conditions are satisfied: (i) no power $\alpha^{s}, s \geq 1$, of $\alpha$ is inner; (ii) the only ideals of $R$ invariant under $\alpha$ are 0 and $R$. These conditions are also known to be necessary and sufficient for the skew Laurent polynomial ring $R\left[x, x^{-1}, \alpha\right]$ to be simple [9]. The object of this paper is to find conditions which are sufficient for $R\left[x, x^{-1}, \alpha\right]$ to be primitive. The results obtained are remarkably similar to those of [8]. Two logically independent conditions are each found to be sufficient for the primitivity of $R\left[x, x^{-1}, \alpha\right]$. Of these, one is also shown to be sufficient for $R[x, \alpha]$ to be primitive. Included in the examples illustrating these results are some applications to the theory of primitive group rings. The basic techniques involved are also applied to produce a counterexample to the converse of a theorem of Goldie and Michler [3] on when $R\left[x, x^{-1}, \alpha\right]$ is a Jacobson ring.

The author wishes to express his thanks to Dr. C. R. Jordan for a number of helpful suggestions during the preparation of this paper.

1. Throughout, $R$ will denote a ring with identity, $\alpha$ will be an automorphism of $R$ and $R\left[x, x^{-1}, \alpha\right]$ will denote the skew Laurent polynomial ring, i.e. the ring of polynomials over $R$ in an indeterminate $x$ and its inverse, with multiplication subject to the relation

$$
x r=\alpha(r) x \text { for all } r \in R .
$$

An ideal $I$ of $R$ is said to be an $\alpha$-ideal of $R$ if $\alpha(I)=I$. An $\alpha$-ideal $I$ of $R$ is said to be $\alpha$-prime if for all $\alpha$-ideals $A, B$ of $R, A B \subseteq I$ implies $A \subseteq I$ or $B \subseteq I$. $R$ is said to be $\alpha$-prime if the ideal 0 is $\alpha$-prime.

The following result is easily proved.

Proposrrion 1 (cf. [3, Lemmas 1.1, 1.3, 1.7], [7, Lemmas 1.3, 1.4]). Let $S=$ $R\left[x, x^{-1}, \alpha\right]$, let $I$ be an ideal of $S$ and let $J$ be an $\alpha$-ideal of $R$. Then

(i) $I \cap R$ is an $\alpha$-ideal of $R$ and $J S$ is an ideal of $S$;

(ii) $\frac{S}{(I \cap R) S} \simeq \frac{R}{I \cap R}\left[x, x^{-1}, \alpha\right]$;

(iii) if $I$ is prime then $I \cap R$ is $\alpha$-prime and if $J$ is $\alpha$-prime then $J S$ is prime.

For any ring $T$ the Jacobson radical of $T$ is denoted $J(T)$. If $I$ is an ideal of $T$, we denote by $J(I)$ the ideal of $T$ such that $J(I) / I=J(T / I) . \mathscr{C}_{T}(I)$ will denote the set $\{c \in T:[c+I]$ is a regular element of $T / \Gamma$.

Proposition 2. Let $S=R\left[x, x^{-1}, \alpha\right]$. If $R$ is right noetherian and $\alpha$-prime then $J(S)=0$.

Glasgow Math. J. 19 (1978) 79-85. 
Proof. This follows from [6, Theorem 2].

\section{2. $\alpha$-primitive rings and $\alpha \mathbf{G}$ rings.}

DEFINTIION. Let $S=R\left[x, x^{-1}, \alpha\right]$ and let $f(x)=\sum_{i=m}^{n} a_{i} x^{i} \in S$, with $a_{n} \neq 0$ and $a_{m} \neq 0$. Then the length of $f(x)$ is the non-negative integer $n-m$.

Defintrion. The automorphism $\alpha$ is said to be stiff on $R$ if for all non-zero ideals $I$ of $R\left[x, x^{-1}, \alpha\right], I \cap R \neq 0$.

Definition. The automorphism $\alpha$ is said to be rigid on $R$ if the mapping $\theta$ from the set of ideals of $R\left[x, x^{-1}, \alpha\right]$ to the set of $\alpha$-ideals of $R$ defined by $\theta(I)=I \cap R$, for all ideals $I$ of $R\left[x, x^{-1}, \alpha\right]$, is a bijection.

LEMMA 1. (i) If there exists a central element $z$ of $R$ such that $\left(\alpha^{n}(z)-z\right) \in \mathscr{C}_{R}(0)$ for all $n>0$ then $\alpha$ is stiff on $R$;

(ii) if there exists a central element $z$ of $R$ such that $\left(\alpha^{n}(z)-z\right)$ is a unit for all $n>0$ then $\alpha$ is rigid on $R$.

Proof. (i) Let $I$ be a non-zero ideal of $S$. Let $f(x)$ be a non-zero element of $I$ of minimal length, $f(x)=\sum_{i=m}^{n} a_{i} x^{i}, a_{m} \neq 0, a_{n} \neq 0$. Since $f(x) x^{r} \in I$ for all integers $r$, it is clear that $m$ may be assumed to be 0 . Suppose that $n>0$. Let $g(x)=f(x) z-z f(x)=\sum_{i=0}^{n} a_{i}\left(\alpha^{i}(z)-z\right) x^{i}$. By the choice of $z, g(x) \neq 0$ but $g(x) \in I$ and the length of $g(x)$ is less than the length of $f(x)$, which contradicts the choice of $f(x)$. Hence $n=0$ and $0 \neq f(x)=a_{0} \in R$. Thus $\alpha$ is stiff on $R$.

(ii) It is sufficient to show that $I=(I \cap R) S$ for all ideals $I$ of $S$, where $S=$ $R\left[x, x^{-1}, \alpha\right]$. If $I$ is an ideal of $S$ then, by (i), $\alpha$ is stiff on $R /(I \cap R)$. It follows from Proposition 1(ii) that $I=(I \cap R) S$.

Defintron. $R$ is said to be $\alpha$-primitive if there exists a maximal right ideal $M$ of $R$ such that $M$ contains no non-zero $\alpha$-ideals of $R$.

THEOREM 1 (cf. [8, Theorem 1]. If $R$ is $\alpha$-primitive and $\alpha$ is stiff on $R$ then $R\left[x, x^{-1}, \alpha\right]$ is primitive.

Proof. The proof is a precise analogue of that of [8, Theorem 1].

Defintion. $R$ is said to be $\alpha G$ if it is $\alpha$-prime and the intersection of the non-zero $a$-prime ideals of $R$ is non-zero.

THEOREM 2 (cf. [8, Theorem 2]). If $R$ is right noetherian, $R$ is $\alpha G$ and $\alpha$ is stiff on $R$ then $R\left[x, x^{-1}, \alpha\right]$ is primitive.

Proof. Let $I$ denote the intersection of the non-zero $\alpha$-prime ideals of $R$ and let $P$ be a non-zero primitive ideal of $S$, where $S=R\left[x, x^{-1}, \alpha\right]$. Since $\alpha$ is stiff, it follows from 
Proposition 1 that $I \subseteq P \cap R$. Consequently either $S$ is primitive or $I \subseteq J(S)$. But $I \neq 0$ and $J(S)=0$ by Proposition 2 . Hence $S$ is primitive.

If $D$ is a division ring which is not algebraic over its centre then the ordinary Laurent polynomial ring $D\left[x, x^{-1}\right]$ is primitive. Consequently the condition that $\alpha$ is stiff on $R$ is not necessary for $R\left[x, x^{-1}, \alpha\right]$ to be primitive. Example 1 (resp. Example 2) below is of a ring $R$ with automorphism $\alpha$ satisfying the conditions of Theorem 2 (resp. Theorem 1) but not those of Theorem 1 (resp. Theorem 2). Thus the statements " $R$ is $\alpha G$ " and " $R$ is $\alpha$-primitive" are logically independent and neither is necessary for $R\left[x, x^{-1}, \alpha\right]$ to be primitive.

Example 1 (cf. [8, Example 1]). Let $R=k[[y]]$ be the power series ring over a field $k$ of characteristic 0 . Let $\alpha$ be the $k$-automorphism of $R$ such that $\alpha(y)=2 y$. The non-zero ideals of $R$ are of the form $y^{r} R, r>0$, and are all $\alpha$-invariant. It follows that the only non-zero $\alpha$-prime ideal of $R$ is $y R$ and hence that $R$ is $\alpha \mathrm{G}$. For $n>0, \alpha^{n}(y)-y=$ $\left(2^{n}-1\right) y \in \mathscr{C}_{R}(0)$ and so, by Lemma $1(i), \alpha$ is stiff on $R$. It follows from Theorem 2 that $R\left[x, x^{-1}, \alpha\right]$ is primitive. However, the only maximal ideal of the commutative ring $R$ is an $\alpha$-ideal, so that $R$ is not $\alpha$-primitive.

EXAMPLE 2 (cf. [8, Example 2]). Let $R=k(t)[y]$ be the polynomial ring in an indeterminate $y$ over the field of rational functions in an indeterminate $t$ over a field $k$ of characteristic 0 . Let $\alpha$ be the $k$-automorphism such that $\alpha(t)=2 t$ and $\alpha(y)=2 y$. Let $M$ be the maximal ideal $(y-1) R$. Then for all integers $i, \alpha^{i}(M)=\left(2^{i} y-1\right) R$, so that $\bigcap_{i=-\infty}^{\infty} \alpha^{i}(M)=0$ and $M$ contains no non-zero $\alpha$-ideals. Thus $R$ is $\alpha$-primitive. For $n>0$, $\alpha^{n}(y)-y=\left(2^{n}-1\right) y \in \mathscr{C}_{R}(0)$ and so, by Lemma $1(i), \alpha$ is stiff on $R$. It follows from Theorem 1 that $R\left[x, x^{-1}, \alpha\right]$ is primitive. $R$ is not $\alpha \mathrm{G}$ because for all $\lambda \in k,(y-\lambda t) R$ is a non-zero $\alpha$-prime ideal and $\bigcap_{\lambda \in k}(y-\lambda t) R=0$ since $k$ is infinite.

ExAmple 3 (cf. [8, Example 3]). Let $R=k[y]$ be the polynomial ring over a field $k$ of characteristic 0 and $\alpha$ the $k$-automorphism of $R$ such that $\alpha(y)=2 y . R$ is $\alpha$-primitive because $\bigcap_{i=-\infty}^{\infty} \alpha^{i}((y-1) R)=0$ as in Example 2. $R$ is $\alpha \mathrm{G}$ because the only non-zero $\alpha$-ideals of $R$ are those of the form $y^{r} R, r>0$, so that $y R$ is the only non-zero $\alpha$-prime ideal of $R$. As in the previous examples $\alpha$ is stiff on $R$, so that, by Theorem 1 or Theorem $2, R\left[x, x^{-1}, \alpha\right]$ is primitive.

3. Primitivity of $R[x, \alpha]$. The object of this section is to show that $R[x, \alpha]$ is primitive whenever the conditions of Theorem 2 hold. By adapting the argument given in $[5, p .22]$ for the case where $R$ is a field it is easy to prove the following. 
Proposition 3 [9]. If the only $\alpha$-ideals of $R$ are 0 and $R$ and if $\alpha^{n}$ is an outer automorphism for all $n>0$, then:

(i) every ideal of $R[x, \alpha]$ contains a power of $x$;

(ii) $R[x, \alpha]$ is primitive;

(iii) $R\left[x, x^{-1}, \alpha\right]$ is simple.

Lemma 2. If there exists a central element $z$ of $R$ such that $\alpha^{n}(z)-z \in \mathscr{C}_{R}(0)$ for all $n>0$ then for all non-zero prime ideals $P$ of $R\lfloor x, \alpha]$ either $x \in P$ or $P \cap R$ is a non-zero $\alpha$-prime ideal of $R$.

Proof. Let $P$ be a non-zero prime ideal of $R[x, \alpha]$ such that $x \notin P$ and let $f(x)=$ $a_{n} x^{n}+\ldots+a_{1} x+a_{0}$ be a non-zero element of minimal degree in $P$. By [3, Lemma 1.2], $x \in \mathscr{C}_{R[x, \alpha]}(P)$ so that $a_{0} \neq 0$. The argument of Lemma 1(i) now shows that $f(x)=a_{0} \in$ $P \cap R$ so that $P \cap R \neq 0$. Finally $P \cap R$ is $\alpha$-prime by [3, Lemmas 1.2,1.3].

Theorem 3. If $R$ is right noetherian, $R$ is $\alpha G$, and $\left(\alpha^{n}(z)-z\right) \in \mathscr{C}_{R}(0)$ for some central $z \in R$ and all $n>0$, then $R[x, \alpha]$ is primitive.

Proof. Let $I$ denote the intersection of the non-zero $\alpha$-prime ideals of $R$. Suppose that $R[x, \alpha]$ is not primitive and let $P$ be a primitive ideal of $R[x, \alpha]$. If $x \notin P$ then, by Lemma $2, P \cap R$ is a non-zero $\alpha$-prime ideal of $R$ and hence $I \subseteq P \cap R$. It follows that $0 \neq I x \subseteq J(R[x, \alpha])$. But by [6, Theorem 2], $J(R[x, \alpha])=0$. Thus $R[x, \alpha]$ is primitive.

If $R$ and $\alpha$ are as in Example 2 or Example 3 then the hypotheses of Theorem 3 are satisfied and $R[x, \alpha]$ is primitive. In neither of these examples are the conditions of Proposition 3 satisfied. The next example is of a ring $R$ and automorphism $\alpha$ satisfying the conditions of Proposition 3 but not those of Theorem 3.

EXAMPLE 4. Let $K=k(t)$ be the field of rational functions over a field $k$ of characteristic 0 . Let $\delta$ be the derivation $d / d t$ and let $R$ be the Ore extension $K[y, \delta] . R$ is known to be simple, see e.g. [2, Theorem 3.2], and clearly $K \backslash\{0\}$ is the set of units of $R$. Let $\alpha$ be the $k$-automorphism of $K$ such that $\alpha(t)=t+1$. Extend the action of $\delta$ and $\alpha$ to $R$ by setting $\delta(y)=0$ and $\alpha(y)=y$. Since $\delta \alpha=\alpha \delta, \alpha$ is then an automorphism of $R$. To see that $\alpha^{n}$ is outer for all $n>0$ let $c \in K \backslash\{0\}$ and let $\beta$ be the inner automorphism, $\beta(r)=c^{-1} r c$ for all $r \in R$. In particular $\beta(y)=c^{-1} y c=y+c^{-1} \delta(c)$. Since for $n>0, \alpha^{n}(y)=y+n$ and there does not exist $c \in K \backslash\{0\}$ such that $\delta(c)=n c$, it follows that $\alpha^{n}$ is outer for all $n>0$. $R$ is simple and so, by Proposition 3 (ii), $R[x, \alpha]$ is primitive. However, the conditions of Theorem 3 are not satisfied since the centre of $R$ is $k$ and $\alpha$ acts as the identity on $k$. By Proposition 3(ii), $R\left[x, x^{-1}, \alpha\right]$ is simple and hence $\alpha$ is stiff on $R$. It follows that the converse of Lemma 1.1 (i) is false.

4. Application to group rings. Let $k$ be a field and $G$ a group having a normal subgroup $H$ such that $G / H$ is an infinite cyclic group, generated by $x H$, say. Let $\alpha$ be the $k$-automorphism of the group ring $k H$ defined by setting $\alpha(h)=x h x^{-1}$ for all $h \in H$. Then $k G \simeq k H\left[x, x^{-1}, \alpha\right]$ and the results of $\$ 2$ apply. In particular we have the following result, where for $h \in G, C_{G}(h)=\{g \in G: h g=g h\}$. 
TheOREM 4. Let $k, G, H$ and $\alpha$ be as above. If $k H$ is prime and $\alpha$-primitive, and there exists $h \in H$ such that $C_{G}(h)=H$ then $k G$ is primitive.

Proof. For $n \geq 1, x \notin C_{G}(h)$, so $\alpha^{n}(h)-h \neq 0$. Since $h$ is central in $H$ and $\alpha$ is an automorphism, $\alpha^{n}(h)-h$ is central and hence, since $k H$ is prime, $\alpha^{n}(h)-h \in \mathscr{C}_{k H}(0)$. By Lemma $1(i), \alpha$ is stiff on $k H$. It follows by Theorem 1 and the above remarks that $k G$ is primitive.

ExAmple 5. Let $G=C_{\infty} \sim C_{\infty}$ be the restricted wreath product of two infinite cyclic groups. $G$ is a cyclic extension of $H$ where $H$ is the restricted direct product of a countable number of infinite cyclic groups. For a given field $k, k H$ is then the Laurent polynomial ring over $k$ in a countable set of commuting indeterminates $\left\{x_{i}\right\}_{i \in z}$. If $\alpha$ is the $k$-automorphism of $k H$ such that $\alpha\left(x_{i}\right)=x_{i+1}$ for all $i \in \mathbb{Z}$ then $k G=k H\left[x, x^{-1}, \alpha\right]$. We claim that $k G$ is primitive for all fields $k$.

Consider first the case where $k$ is countable, possibly finite. It is clear that any non-zero $\alpha$-ideal $I$ of $k H$ has non-zero intersection with $k\left[x_{1}, x_{2}, \ldots, x_{n}\right]$ for some $n=n(I)$. Let $\hat{k}$ denote the algebraic closure of $k$. Then for each $r \geq 1$ the set of $r$-tuples $\left(\lambda_{1}, \lambda_{2}, \ldots, \lambda_{r}\right), \lambda_{i} \in \hat{k} \backslash\{0\}$ is countable. The union of these sets, taken over all $r \geq 1$, is countable and hence there exists a sequence $(\mu)_{i \geq 1}, \mu_{i} \in \hat{k} \backslash\{0\}$, such that for every positive integer $r$ and $r$-tuple $\left(\lambda_{1}, \lambda_{2}, \ldots, \lambda_{r}\right), \lambda_{i} \in \hat{k} \backslash\{0\}$, there exists $l \geq 1$ such that $\lambda_{1}=\mu_{1}, \lambda_{2}=\mu_{l+1}, \ldots, \lambda_{r}=\mu_{i+r+1}$. For $i \leq 0$ let $\mu_{i}=1$. Let $M=\sum_{i \leq j} M_{i, j} k H$, where for the integers $i, j$ such that $i \leq j, M_{i, j}=\left\{f \in k\left[x_{i}, \ldots, x_{j}\right]: f\left(\mu_{i}, \ldots, \mu_{j}\right)=0\right\}$. By Hilbert's Nullstellensatz [1, Proposition 2, p. 351], each $M_{i, j}$ is a maximal ideal of $k\left[x_{i}, \ldots, x_{j}\right]$ and it follows that $M$ is a maximal ideal of $k H$. Suppose that there exists a non-zero $\alpha$-ideal $I$ of $k H$ such that $I \subseteq M$. Then for some positive integer $n$ there exists non-zero $f=$ $f\left(x_{1}, x_{2}, \ldots, x_{n}\right) \in I \cap k\left[x_{1}, x_{2}, \ldots, x_{n}\right]$. Since $\alpha^{i}(f) \in M$ for all $i, \alpha^{i}(f) \in M_{i, n+i-1}$ for all $i$ and hence

$$
\alpha^{i}(f)\left(\mu_{i}, \ldots, \mu_{n+i-1}\right)=0 \text { for all } i
$$

Equivalently,

$$
f\left(\mu_{i}, \ldots, \mu_{n+i-1}\right)=0 \text { for all } i \text {. }
$$

Now let $N$ be any maximal ideal of $k\left[x_{1}, x_{2}, \ldots, x_{n}\right]$. By the Nullstellensatz, either $x_{1} x_{2} \ldots x_{n} \in N$ or there exists an $n$-tuple $\left(\lambda_{1}, \lambda_{2}, \ldots, \lambda_{n}\right), \lambda_{i} \in \hat{k} \backslash\{0\}$, such that, for $g \in k\left[x_{1}, x_{2}, \ldots, x_{n}\right], g \in N$ iff $g\left(\lambda_{1}, \lambda_{2}, \ldots, \lambda_{n}\right)=0$. In the latter case there exists $l \geq 1$ such that $\lambda_{1}=\mu_{l}, \lambda_{2}=\mu_{l+1}, \ldots, \lambda_{n}=\mu_{l+n-1}$ so that by (1), $f \in N$. It follows that $0 \neq x_{1} x_{2} \ldots x_{n} f \in N$ for every maximal ideal $N$ of $k\left[x_{1}, x_{2}, \ldots, x_{n}\right]$. But the Jacobson radical of $K\left[x_{1}, x_{2}, \ldots, x_{n}\right]$ is zero, which gives a contradiction. Thus $M$ contains no non-zero $\alpha$-ideal of $k H$ and $k H$ is $\alpha$-primitive. Since $k H$ is a commutative domain and $C_{G}\left(x_{1}\right)=H$, it follows from Theorem 4 that $k G$ is primitive

Now let $k$ be an arbitrary field and $l$ the prime subfield of $k$. Then $l G$ is primitive by the above. It follows from [10, Theorem 2] that $k G$ is primitive. We note that for the case 
where the transcendence degree of $k$ over $l$ is infinite $k G$ is known to be primitive by $[\mathbf{1 0}$, Corollary 13].

EXAmple 6. Let $H=\left\langle x_{1}, x_{2}\right\rangle$ be a free abelian group of rank 2 and let $\alpha$ be the automorphism of $H$ such that $\alpha\left(x_{1}\right)=x_{2}$ and $\alpha\left(x_{2}\right)=x_{1} x_{2}$. Let $G$ be the semidirect product $H \times{ }_{\alpha}\langle x\rangle$, where $\langle x\rangle$ is infinite cyclic. We claim that if $k$ is a field of characteristic zero then $k H$ is $\alpha$-primitive and hence that $k G$ is primitive.

Consider first the case where $k=\mathbb{Q}$, the field of rational numbers. Let $M=$ $\left(x_{1}-2\right) \mathbb{Q} H+\left(x_{2}-2\right) \mathbb{Q} H$, a maximal ideal of $\mathbb{Q} H$. Suppose that there exists a non-zero $\alpha$-ideal $I$ of $\mathbb{Q} H$ such that $I \subseteq M$. Then there exists non-zero $f\left(x_{1}, x_{2}\right) \in \mathbb{Q}\left[x_{1}, x_{2}\right]$ such that $\alpha^{n}\left(f\left(x_{1}, x_{2}\right)\right) \in M$ for all $n \geq 0$, i.e. $f\left(\alpha^{n}\left(x_{1}\right), \alpha^{n}\left(x_{2}\right)\right)=0$ for all $n \geq 0$. In general, $\alpha^{n}\left(x_{1}\right)=$ $x_{1}^{u(n-1)} x_{2}^{u(n)}$ and $\alpha^{n}\left(x_{2}\right)=x_{1}^{u(n)} x_{2}^{u(n+1)}$, where $u(0)=0$ and, for $i \geq 1, u(i)$ is the $i$ th Fibonacci number. Thus $f\left(2^{u(n-1)} 2^{u(n)}, 2^{u(n)} 2^{u(n+1)}\right)=0$ for all $n \geq 0$, i.e. $f\left(2^{u(n+1)}, 2^{u(n+2)}\right)=$ 0 for all $n \geq 0$. It follows from Lemma 3 below that $f=0$, which gives a contradiction. Thus $\mathbb{Q} H$ is $\alpha$-primitive, and since $\mathbb{Q} H$ is a commutative domain and $C_{G}\left(x_{1}\right)=H$ it follows from Theorem 3 that $\mathbb{Q} G$ is primitive. Since $\left\{g \in G:\left\{y^{-1} g y: y \in G\right\}\right.$ is finite $\}=\{1\}$ it follows from [10, Theorem 2] that $k G$ is primitive for all fields $k$ of characteristic 0 .

Lemma 3. For $i \geq 1$ let $u(i)$ denote the $i$-th Fibonacci number and let $f=f\left(x_{1}, x_{2}\right) \in$ $\mathbb{Q}\left[x_{1}, x_{2}\right]$ be such that $f\left(2^{u(n)}, 2^{u(n+1)}\right)=0$ for all $n \geq 1$. Then $f=0$.

Proof. For $n \geq 1$ let $\lambda(n)=u(n+1) / u(n)$. It is known that $\lambda(n) \rightarrow \lambda=(1+\sqrt{ } 5) / 2$ as $n \rightarrow \infty$ (see e.g. [4, Chapter X]). Define an order $>$ on the set of monomials $x_{1}^{p} x_{2}^{q}, p, q \geq 0$ as follows:

$$
x_{1}^{p} x_{2}^{q}>x_{1}^{r} x_{2}^{s} \text { iff } p+\lambda q>r+\lambda s .
$$

Since $\lambda$ is irrational, $>$ is a total order. Suppose $f \neq 0$. Then for some integer $t>1$

$$
f\left(x_{1}, x_{2}\right)=\sum_{i=1}^{t} f_{i} x_{1}^{p(i)} x_{2}^{q(i)}
$$

where $f_{i} \in \mathbb{Q} \backslash\{0\}$ for $1 \leq i \leq t$ and $x_{1}^{p(i)} x_{2}^{q(i)}<x_{1}^{p(j)} x_{2}^{q(j)}$ whenever $1 \leq i<j \leq t$. For $n \geq 1$,

$$
\begin{aligned}
f\left(2^{u(n)}, 2^{u(n+1)}\right) & =\sum_{i=1}^{t} f_{i} 2^{p(i) u(n)+q(i) u(n+1)} \\
& =\sum_{i=1}^{t} f_{i} 2^{u(n)(p(i)+\lambda(n) q(i))} \\
& =f_{t} 2^{u(n)(p(t)+\lambda(n) q(t))}\left(1+\sum_{i=1}^{t-1}\left(f_{i} / f_{t}\right) 2^{u(n)(p(i)+\lambda(n) q(i)-(p(t)+\lambda(n) q(t)))}\right) .
\end{aligned}
$$

But $p(i)+\lambda q(i)<p(t)+\lambda q(t)$ for $1 \leq i \leq t-1$ and, as $n \rightarrow \infty, \lambda(n) \rightarrow \lambda$ and $u(n) \rightarrow \infty$. Hence

$$
\sum_{i=1}^{t-1}\left(f_{i} / f_{t}\right) 2^{u(n)(p(i)+\lambda(n) q(i)-(p(t)+\lambda(n) q(t)))} \rightarrow 0 \quad \text { as } n \rightarrow \infty .
$$

It follows from (2) that there exists $N$ such that for all $n \geq N, f\left(2^{u(n)}, 2^{u(n+1)}\right) \neq 0$. 
5. Jacobson rings. A ring $R$ is said to be a Jacobson ring if every prime ideal is the intersection of primitive ideals. Goldie and Michler [3, Theorem 1.12] have shown that if $\alpha$ is an automorphism of a right noetherian Jacobson ring $R$ then $R\left[x, x^{-1}, \alpha\right]$ is also a right noetherian Jacobson ring. Using the ideas of $\$ 2$ we prove the following result, providing counterexamples to the converse of [3, Theorem 1.12].

THEOREM 5. Let $R$ be right noetherian. If $\alpha$ is rigid on $R$ then $S=R\left[x, x^{-1}, \alpha\right]$ is a Jacobson ring.

Proof. Let $P$ be a prime ideal of $S$. Then by Proposition $1, P \cap R$ is $\alpha$-prime and $(P \cap R) S$ is an ideal of $S$. But $\alpha$ is rigid and hence $P=(P \cap R) S$. By Proposition 1 (ii),

$$
\frac{S}{P}=\frac{S}{(P \cap R) S} \simeq \frac{R}{P \cap R}\left[x, x^{-1}, \alpha\right] .
$$

It follows by Proposition 2 that $J(P)=P$, i.e. that $P$ is the intersection of primitive ideals. Thus $S$ is a Jacobson ring.

EXAMPLE 7. Let $R=k(t)[[y]]$ be the power series ring in an indeterminate $y$ over the field of rational functions in an indeterminate $t$ over a field $k$ of characteristic 0 . Let $\alpha$ be the $k$-automorphism of $R$ such that $\alpha(t)=2 t$ and $\alpha(y)=y$. Then for $n \geq 1, \alpha^{n}(t)-t=$ $\left(2^{n}-1\right) t$ is a unit and so, by Lemma 1 (ii), $\alpha$ is rigid on $R$. It follows from Theorem 4 that $R\left[x, x^{-1}, \alpha\right]$ is Jacobson. However, since $R$ is a local domain, it is certainly not a Jacobson ring.

\section{REFERENCES}

1. N. Bourbaki, Commutative algebra (Hermann/Addison-Wesley, 1972).

2. J. Cozzens and C. Faith, Simple noetherian rings (Cambridge Univ. Press, 1975).

3. A. W. Goldie and G. Michler, Ore extensions and polycyclic group rings, J. London Math. Soc. (2), 9 (1974), 337-345.

4. G. H. Hardy and E. M. Wright, An introduction to the theory of numbers (4th edition) (Oxford Univ. Press, 1960). 1964).

5. N. Jacobson, Structure of rings (rev. edition) (Amer. Math. Soc. Colloquium Publications,

6. C. R. Jordan and D. A. Jordan, A note on the semiprimitivity of Ore extensions, Comm. Algebra 4 (1976), 647-656.

7. D. A. Jordan, Noetherian Ore extensions and Jacobson rings, J. London Math. Soc. (2), 10 (1975), 281-291.

8. D. A. Jordan, Primitive Ore extensions, Glasgow Math. J. 18 (1977), 93-97.

9. D. A. Jordan, Ph.D. thesis, Univ. of Leeds (1975).

10. D. S. Passman, Primitive group rings, Pacific J. Math. 47 (1973), 499-506.

Department of Pure Mathematics

UNIVERSITY OF SHEFFIELD

SHEFFIELD

S10 2TN 